\title{
Chest Wall PNET Masquerading as Breast Lump: A Case Report with Diagnostic Challenge
}

\author{
Vachaspati Kumar Mishra ${ }^{1}$, Ajeet Kumar Gandhi ${ }^{1}$, Madhup Rastogi ${ }^{1}$, Satyajeet Rath ${ }^{1}$, Akash Agarwal2, \\ Ayushi Patni ${ }^{1}$, Avinash Poojari ${ }^{1}$, Nuzhat Husain ${ }^{3}$ \\ Departments of ${ }^{1}$ Radiation Oncology, ${ }^{2}$ Surgical Oncology and ${ }^{3}$ Pathology; Dr. Ram Manohar Lohia Institute of Medical Sciences, \\ Lucknow, Uttar Pradesh, India.
}

\section{Corresponding Author:}

Dr Satyajeet Rath

Email: satyajeetrath@gmail.com

This is an Open Access article distributed under the terms of the Creative Commons Attribution License (creativecommons.org/ licenses/by/3.0).

Received : December 8, 2019

Accepted : January 29, 2020

Published : February 5, 2020

\begin{abstract}
Background: Chest wall primitive neuroectodermal tumor (PNET) is a rare tumor necessitating a prompt diagnosis. Unfortunately, the diagnosis of this tumor can be extremely challenging and misdiagnosis is at times unavoidable. Case Report: Here we present one such rare case of chest wall PNET. Patient initially had complaints of pain and lump in the right breast. Contrast enhanced computed tomography (CECT) of thorax showed large mass with both intra-thoracic and extra-thoracic extension. Trucut biopsy from lump was suggestive of PNET on histopathology and immunohistochemistry. Later patient was treated with induction chemotherapy with alternating VAC/IE followed by right chest wall resection. Post-operative histopathology was suggestive of complete pathological response. ${ }^{18}$ FDG PET-CT done subsequently was suggestive of complete metabolic response. Conclusion: Chest wall PNETs may mimic as breast lump, posing diagnostic dilemma. Multimodality treatment may yield excellent outcome as in our case.
\end{abstract}

Keywords: Biopsy, Diagnostic Errors, Thoracic Wall, Neuroectodermal Tumors, Pain.

\section{Introduction}

Primitive neuroectodermal tumor (PNET) is a rare undifferentiated and aggressive small, round cell tumor that carries identical chromosomal translocation $\mathrm{t}(11 ; 22)$ as Ewing's sarcoma (ES) classified under Ewing's family of tumors (EFTs) [1]. The incidence of chest wall PNET is more common in age group less than 20 years and has likely been underreported in literature as techniques like immunohistochemistry and cytogenetics are recent advancements [2]. Here, we report a rare case of PNET of chest wall mimicking breast lump and presenting as a diagnostic dilemma.

\section{Case Report}

A 24 years old pre-menopausal female patient with no medical risk factors presented in surgery clinic with pain and lump in the right breast for two weeks. After initial clinical examination high resolution ultrasonogram (HRUSG) of the right breast showed $45 \times 23 \times 34 \mathrm{~mm}$ ill-defined collection in right breast extending to thoracic cavity suggestive of empyema necessitans and was prescribed antibiotics for two weeks. The lump did not resolve, sonomammography performed after 4 weeks revealed a persistent echopenic mass lesion of size $30 \times 25 \times 15 \mathrm{~mm}$ with cystic areas. Excisional biopsy was performed and histopathology was reported as inflammatory pseudotumor. Immunohistochemistry was suggestive of vimentinpositive and (CD-34, SMA, S-100, ALK, CK, LCA-negative) and Ki-67 index was less than 5\%. Patient was given symptomatic medications. Pain was relieved, but the mass lesion was increasing in size in the right breast for about 3 months after which repeat sonomammography showed a large echogenic mass lesion with heterogenous texture 
of $91.8 \times 87.9 \times 78.8 \mathrm{~mm}$ with cystic areas in right breast and no axillary lymphadenopathy. Core biopsy from right breast lump was suggestive of fibroadenosis.

Patient presented to us at this point of time. Contrast enhanced computed tomography (CECT) of thorax was suggestive of $169 \times 165 \times 156 \mathrm{~mm}$ mass with intra-thoracic component extending to mediastinum and lateral chest wall component infiltrating the right breast parenchyma; right $5^{\text {th }}$ rib expansion was present with no mediastinal lymphadenopathy [Fig.1]. Trucut biopsy from chest wall lesion was suggestive of PNET on histopathology and on immunohistochemistry it was CD-99, FLI-1 and S-100 were positive and Desmin and Pan-CK negative [Fig.2]. Bone scan revealed destruction of right $5^{\text {th }}$ rib and was negative for distant skeletal metastasis. Bone marrow aspiration and biopsy were normal. Patient was given chemotherapy with alternating VAC (vincristine $2 \mathrm{mg} / \mathrm{m}^{2}$, maximum dose $2 \mathrm{mg}$ ), doxorubicin $75 \mathrm{mg} / \mathrm{m}^{2}$ and cyclophosphamide $1.2 \mathrm{gm} / \mathrm{m}^{2}$ on day $1 \mathrm{q} 3$ weekly) and IE (ifosfamide $1.8 \mathrm{gm} / \mathrm{m}^{2}$ and etoposide $100 \mathrm{mg} / \mathrm{m}^{2}$ for 5 days q 3 weekly) for 12 weeks. CECT thorax repeated after 12 weeks of chemotherapy revealed significant reduction in size of the primary lesion (both intra-thoracic and extra-thoracic components to $88 \times 55 \times 84 \mathrm{~mm}$ ) [Fig.3]. Then patient was discussed in multidisciplinary tumor board and was advised for further one cycle of chemotherapy with alternative VAC/IE regimen and assessment for surgery. Patient underwent chest wall resection $\left(4^{\text {th }}-6^{\text {th }}\right.$ rib $)$. Post-operative histopathology was suggestive of complete pathological response. The patient further received four more cycles of chemotherapy with alternative VAC/IE. Doxorubicin was replaced with dactinomycin after cumulative dose of $375 \mathrm{mg} / \mathrm{m}^{2}$. ${ }^{18} \mathrm{FDG}$ PET-CT done subsequently was suggestive of complete metabolic response [Fig.4,5].

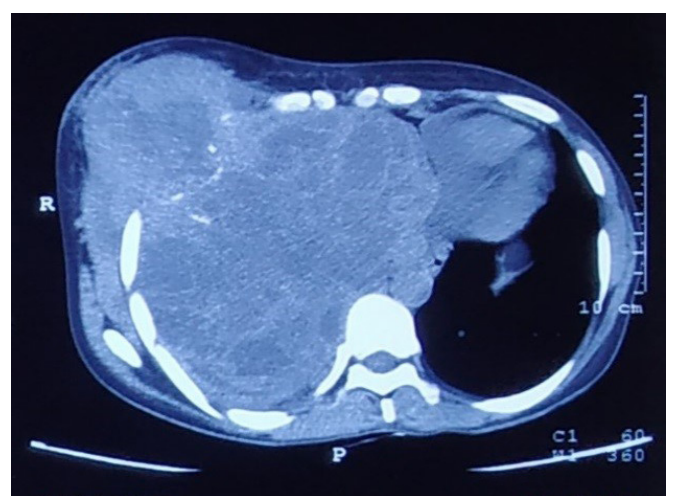

Fig.1: CECT thorax before chemotherapy showing huge mass with both intra-thoracic and extra-thoracic extension.

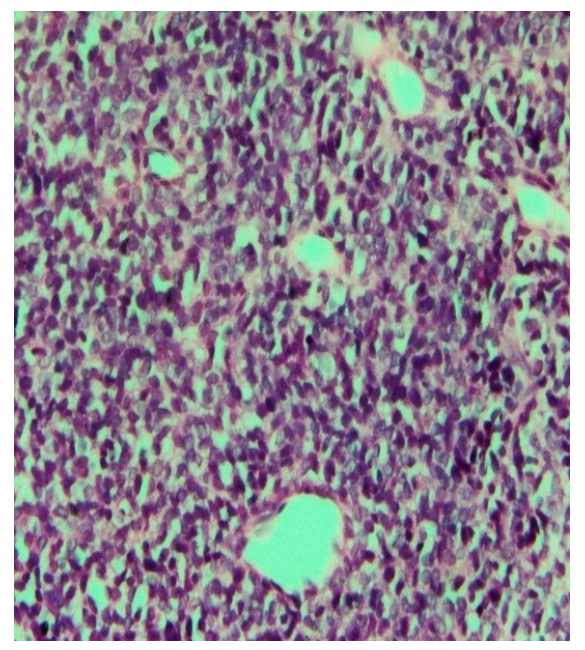

Fig.2: HPE showing small round blue cells, PNET.

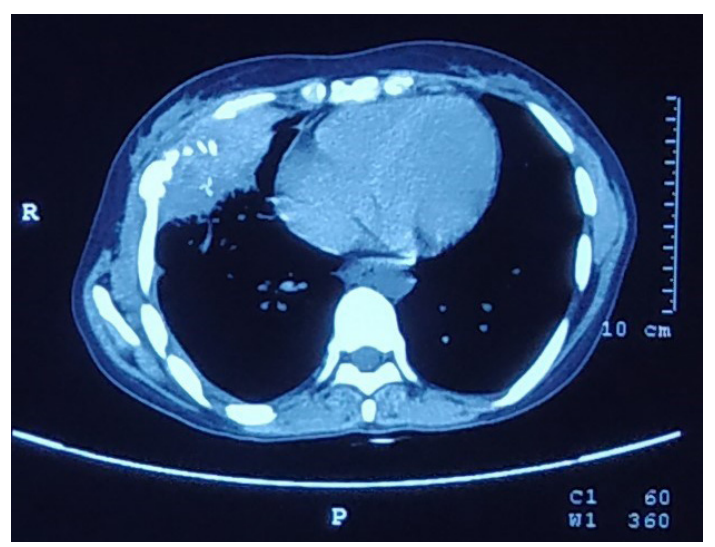

Fig.3: CECT thorax post-chemotherapy before surgery showing significant reduction in size of mass. 


\section{Discussion}

PNET are small round blue cell tumors. These tumors are rare and are malignant in nature. They arise from primitive nerve cells of nervous system but these tumors can be found outside the central nervous system in the pelvis, chest wall and extremities. Chest wall PNET is also known as Askin's tumor [3]. It mainly develops from the soft tissue of chest wall, predominantly in paravertebral region. These tumors are more common in Caucasian ethnic group [4]. A reciprocal translocation between chromosomes 11 and 22 in about $85 \%$ of tumors creates a chimeric EWSFLL-1 fusion gene. It may present as a painful mass associated with cough, breathlessness, weight loss, Horner syndrome or lymphadenopathy. The physical finding may include tenderness and palpable mass over tumor site.

The diagnosis is made by core needle biopsy; sometimes open biopsy may be required. Histopathology remains the cornerstone for the diagnosis of PNETs which show islands of anaplastic, small, round blue cells with scant eosinophilic cytoplasm and high mitotic rate. Rarely, these tumor cells can be large with irregular nuclear membrane and prominent nucleoli [5]. PNET cells show membranous expression of CD99 or MIC2 on immunohistochemistry [6]. Antibody against FLI-1 is specific for Ewing's family of tumors (EFT) [7]. Depending upon the degree of neuroectoderm differentiation, the tumor cells may also express neuron-specific enolase (NSE), synaptophysin and S-100 protein. New potential immunohistochemical marker for EFT is NKX2.2, protein product of the NKX2-2 gene [8]. Cytogenetic studies are important for chromosomal translocation $\mathrm{t}(11 ; 22)$ identification. In chest wall PNETs, the commonest radiological finding is soft tissue density mass which might be associated with erosion of ribs or fluid in pleural cavity [9]. CT thorax have diagnostic role in confirming the solid chest wall mass and intra-thoracic or lung
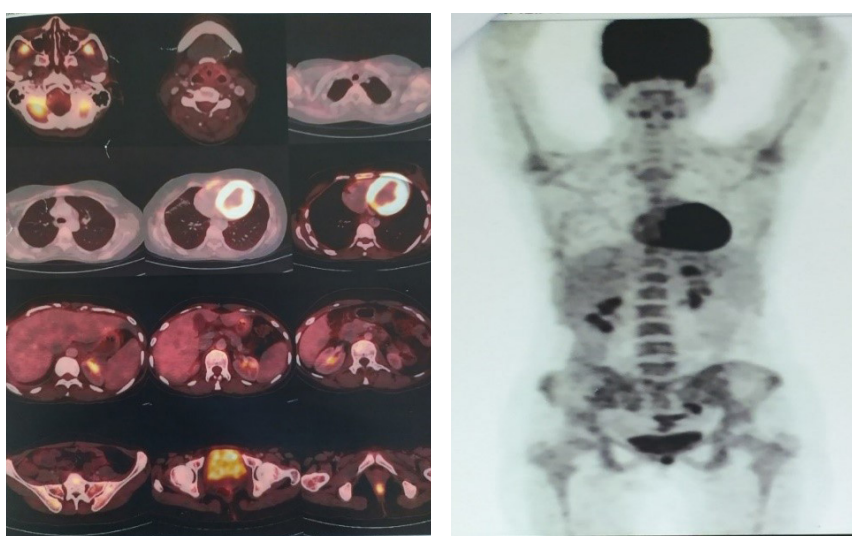

Fig.4,5: PET- CT whole body showing no disease in thorax.

involvement. MRI may also be useful in diagnosing soft tissue involvement. Bone scan is an important investigation as it gives us information about local bone destruction and distant skeletal metastasis.

Patient of chest wall PNETs should be evaluated and treated at tertiary cancer centre as it is must to have multidisciplinary expertise. The two major types of PNETs are localised disease and metastatic disease. PNET has a multi-modality management. Treatment of PNETs depends on stage of disease. Treatment involves surgical resection, neo-adjuvant and adjuvant chemotherapy and radiation [10]. The current standard of care is initial cytoreduction with chemotherapy to deal with micro-metastatic disease and to facilitate effective control measures with wide negative margin. Afterwards definitive radiotherapy or surgery is done to eradicate the primary and consolidation chemotherapy is given to remove occult residual disease. For non-metastatic disease, the chemotherapy protocol followed is alternate courses of VAC with courses of IE every three weekly [11] for 49 weeks; dose is vincristine $2 \mathrm{mg} / \mathrm{m}^{2}$ (maximum dose $2 \mathrm{mg}$ ), doxorubicin 75 $\mathrm{mg} / \mathrm{m}^{2}$ and cyclophosphamide $1.2 \mathrm{gm} / \mathrm{m}^{2}$ and IE, Ifosfamide $1.8 \mathrm{gm} / \mathrm{m}^{2}$ and etoposide $100 \mathrm{mg} / \mathrm{m}^{2}$. Local therapy may be given at 9-12 weeks. Local therapy includes radiotherapy or surgery. Radiotherapy (RT) is aimed to eradicate all disease. RT dose of 40 to 50 Gy to entire bone with an 
additional 10 to $15 \mathrm{~Gy}$ cone down to affected site will yield good result. Indications of post-operative RT are gross or microscopic positive margin, poor histologic response to chemotherapy and pretreatment fracture, hematoma, tissue violation [12]. Post-treatment response assessment is commonly done by ${ }^{18}$ FDG PET-CT. Multimodality treatment may improve the outcome.

\section{Conclusion}

Chest wall PNETs are rare tumors. Diagnosis and treatment require involvement of multimodality team. Chest wall PNETs may mimic as breast lump, posing a diagnostic dilemma. Multimodality treatment yielded excellent outcome in our case.

Contributors: VKM: manuscript writing, and literature review and references; AKG, MR, SR, AA, AyP, AvP: critical inputs into the manuscript and patient management; $\mathrm{NH}$ : literature review, and histopathology. SR will act as a study guarantor. All authors approved the final version of this manuscript and are responsible for all aspects of the study.

Funding: None; Competing interests: None stated.

\section{References}

1. Kauer M, Ban J, Kofler R, Walker B, Davis S, Meltzer $\mathrm{P}$, et al. A molecular function map of Ewing's sarcoma. PLoS One. 2009;4:e5415.

2. International Agency for research on cancer. WHO classification of tumors of soft tissue and bone. $4^{\text {th }}$ ed. Geneva:WHO;2013.

3. Askin FB, Rosai J, Sibley RK, Dehner LP, McAlister $\mathrm{WH}$, et al. Malignant small cell tumor of the thoracopulmonary region in childhood: a distinctive clinicopathological entity of uncertain histogenesis. Cancer. 1979;43:2438-2451.

4. Laskar S, Nair C, Mallik S, Bahl G, Pai S, Shet T, et al. Prognostic factors and outcome in Askin-Rosai tumor: a review of 104 patients. Int. J Radiat Oncol Bio Phys. 2011;79:202-207.

5. Nascimento AG, Unii KK, Pritchard DJ, Cooper KL, Dahlin DC. A clinicopathological study of 20 cases of large cell (atypical) Ewing's sarcoma of bone. Am J Surg Pathol. 1980;4:29-36.

6. Khoury JD. Ewing sarcoma family of tumors. Adv Anat Pthol. 2005;12:212-220.

7. Nilsson G, Wang M, Wejde J, Kreicbergs A, Larsson O. Detection of EWS/FLI-1 by immunostaining. An adjunctive tool in diagnosis of Ewing's sarcoma and PNET on cytological samples and paraffin-embedded archival material. Sarcoma. 1999;3:25-32.

8. Roessner A, Mittler U, Rose I, Radig K, Grote H. Pathology of Ewing'sarcoma. Pathologe. 1996;17:6-17.

9. Sabati JM, Franquet T, Parellada JA, Monil JM, Olive E. Malignant neuroectodermal tumor of the chest wall (Askin Tumor). CT and MR finding in eight patients. Clin Radiol. 1994;49:634-638.

10. Veronesi G, Spaggiari L, De Pas T, Soli PG, De Braud F, Catalano GP, et al. Preoperative chemotherapy is essential for conservative surgery of Askin tumors. J Thorac Cardiovasc Surg. 2003;125:428-429.

11. Grier HE, Krailo MD, Tarbell NJ, Link MP, Fryer CJ, Pritchard DJ, et al. Addition of ifosfamide etoposide to standard chemotherapy for Ewing's sarcoma or PNET of bone. NEJM. 2003;348(8):694-701.

12. Laskar S, Mallick I, Gupta T, Muckaden MA. Post operative radiotherapy for Ewing sarcoma: when, how and how much. Pediatr Blood Cancer. 2008;51:575-580. 